\title{
Chondrogenesis of myoblasts in biodegradable poly-lactide-co-glycolide scaffolds
}

\author{
YANGLIN GU $^{1}$, PENG CHEN ${ }^{1}$, YUSHENG YANG ${ }^{1}, \mathrm{KEQIN} \mathrm{SHI}^{1}$, \\ YUBIN WANG ${ }^{2}$, WENHUI ZHU ${ }^{2}$ and GUOXING ZHU ${ }^{1}$ \\ ${ }^{1}$ Department of Orthopedics, Wuxi No. 2 People's Hospital, Jiangsu 214002; ${ }^{2}$ Department of Sports Medicine, \\ Dongfang Hospital Affiliated to Tongji University, Shanghai 200120, P.R. China
}

Received September 4, 2012; Accepted December 11, 2012

DOI: $10.3892 / \mathrm{mmr} .2012 .1240$

\begin{abstract}
Myoblasts are considered to be an alternative cell source for cell-based meniscal repair due to their multiple differentiation potentials. This study addresses the chondrogenic differentiation of myoblasts seeded into poly-lactide-co-glycolide (PLGA) scaffolds following implantation in a subcutaneous pocket of nude mice. Canine myoblasts isolated from a Beagle were expanded and seeded into PLGA scaffolds and cultured in cartilage-derived morphogenetic protein-2 (CDMP-2) and transforming growth factor- $\beta 1$ (TGF- $\beta 1$ )-containing medium for 2 weeks in vitro. The constructs were implanted into a subcutaneous pocket of 24 combined immunodeficiency mice and harvested after 8 and 12 weeks, respectively. Hematoxylin and eosin staining of the sections of the engineered cartilage at 8 and 12 weeks revealed the regeneration of fibrocartilage. Immunohistochemical staining confirmed a similar distribution of collagen type II in the engineered cartilage as the normal meniscus. At 12 weeks, expression of mRNAs for type I collagen, type II collagen and aggrecan was detected by RT-PCR. The compressive moduli of engineered cartilage reached $85.72 \%$ of the normal meniscus at 12 weeks, with a high level of glycosaminoglycan (GAG) content (no statistical difference from normal). Myoblastseeded PLGA scaffolds express a stable chondrogenic phenotype in a heterotopic model of cartilage transplantation and represent a suitable tool for tissue engineering of cartilage.
\end{abstract}

\section{Introduction}

Meniscus has limited potential of self-repair, and menisci injury may lead to long-term degenerative joint changes $(1,2)$. In addition to other therapies, meniscal regeneration using tissue engineering techniques has been attempted, based

Correspondence to: Dr Guoxing Zhu, Department of Orthopedics, Wuxi No. 2 People's Hospital, 68 Zhongshan Road, Wuxi, Jiangsu 214002, P.R. China

E-mail: guoxing.zhu@yahoo.cn

Key words: myoblasts, differentiation, scaffolds, tissue engineering on the loading and culture of suitable cells into appropriate scaffolds $(3,4)$. With regards to the cell source, meniscal chondrocytes, mesenchymal cells and pluripotential fibroblasts have all been identified as possible sources for the repair of meniscal tissue (5-7). Compared with the cell sources mentioned above, myoblasts are a promising source for meniscal engineering, as they are relatively abundant and easily accessible with minimal donor site morbidity. Myoblasts may also be good candidates for tissue engineering as they have a higher cell yield and proliferate rapidly during in vitro expansion (8).

With regards to the scaffold, a variety of natural or artificial biomaterials have been investigated for use in tissue-engineered meniscus. Due to the poor biomechanical properties and the rapid degradation of fibrin and alginate, polymer scaffolds with a stable, biodegradable and permeable pore network were used to support cell attachment, proliferation and nutrient exchange and to provide stability $(5,9)$.

In this study, non-woven PLGA scaffolds were seeded with myoblasts and transplanted into subcutaneous pockets of 24 combined immunodeficiency (SCID) mice. It was hypothesized that such cell-seeded constructs would demonstrate a cartilage-like morphology with expression of chondrocyte-specific molecules, while also conserving sufficient biomechanical characteristics, after 12 weeks in vivo.

\section{Materials and methods}

Isolation, culture and induction of myoblasts. Canine myoblasts were isolated and cultured as previously described (10). Primary cells were seeded on a culture dish at a density of $5 \times 10^{5}$ cells $/ \mathrm{cm}^{2}$ in Dulbecco's modified Eagle's medium (DMEM; Gibco-BRL, Grand Island, NY, USA) containing $10 \%$ fetal bovine serum (FBS; Gibco-BRL), $300 \mu \mathrm{g} / \mathrm{ml}$ of L-glutamine, $50 \mu \mathrm{g} / \mathrm{ml}$ vitamin $\mathrm{C}, 100 \mathrm{U} / \mathrm{ml}$ penicillin $\mathrm{G}, 100 \mu \mathrm{g} / \mathrm{ml}$ streptomycin and amphotericin B $0.25 \mu \mathrm{g} / \mathrm{ml}$ (all from Sigma, St. Louis, MO, USA). After medium change, cultured myoblasts were subjected to chondrogenic induction with culture medium containing $50 \mathrm{ng} / \mathrm{ml}$ CDMP-2 and $20 \mathrm{ng} / \mathrm{ml}$ TGF- $\beta 1$ (Sigma). The myoblasts were cultured at $37^{\circ} \mathrm{C}$ in a humidified atmosphere of $95 \%$ air and $5 \% \mathrm{CO}_{2}$. The medium was changed every third day, and this washed out all non-adherent cells. Cells were subcultured at a density of $1.0 \times 10^{4}$ cells $/ \mathrm{cm}^{2}$ and treated with $0.25 \%$ trypsin 
plus $0.02 \%$ EDTA (Gibco-BRL) when they reached $80 \%$ confluence. The study was approved by the ethics committee of Tongji University, Shanghai, China.

Immunocytochemistry assay of collagen II. To determine the in vitro chondrogenic induction effect, induced myoblasts were examined for type II collagen expression using immunocytochemical staining. Briefly, cells were incubated at $37^{\circ} \mathrm{C}$ for $1 \mathrm{~h}$ with mouse anti-collagen-II monoclonal antibody (IgG1; BD Biosciences Clontech, Franklin Lakes, NJ, USA) diluted in phosphate-buffered saline (PBS, 1:200), followed by incubation with 1:100 diluted horseradish peroxidase (HRP)conjugated anti-mouse antibody (Dako, Carpinteria, CA, USA) for $30 \mathrm{~min}$ and color development with diaminobenzidine tetrahydrochloride (DAB). Normal menisci served as positive controls.

Preparation of PLGA scaffold and cell seeding. A non-woven copolymer scaffold of L-lactide and glycolide (90/10, PLGA) in the form of fibers was generously provided by Shanghai Ju Rui Biomaterials Co., Inc., (China). The scaffolds were cylindrical, with a diameter of $10 \mathrm{~mm}$ and a thickness of $2 \mathrm{~mm}$. The pore sizes of the non-woven fibers were on average $75 \mu \mathrm{m}$, the pore volume accounted for $97 \%$ of the total volume, and the filament diameter was $13 \mu \mathrm{m}$. The PLGA constructs were treated using the low-pressure plasma technique at the end of the production process. A partially ionized gas reacted with the surface of the scaffolds and formed reactive particles. Prior to cell seeding, the scaffolds were immersed in DMEM/F12 medium containing $10 \%(\mathrm{v} / \mathrm{v})$ FBS for $12 \mathrm{~h}$ to enhance cell adhesion onto the scaffold.

Chondrogenically induced myoblasts at passage $3\left(1.5 \times 10^{7}\right.$ in $0.3 \mathrm{ml}$ ) were harvested and placed onto PLGA scaffolds, respectively, to form cell-scaffold constructs and the constructs were cultured at $37^{\circ} \mathrm{C}$ in a humidified atmosphere of $5 \% \mathrm{CO}_{2}$ for $5 \mathrm{~h}$, which allowed the complete adhesion of myoblasts to the scaffold. The cell-PLGA constructs in inductive media were subsequently cultured in vitro for 14 days. Medium was changed three times a week. As an experimental control, scaffolds with non-induced myoblasts were cultured for the same lengths of time.

Surgical procedure. Six-week-old athymic nude mice were used in this study, and were obtained from the Agricultural Institute of Shanghai Jiaotong University, China. Animal care and experimental procedures were in accordance with the guidelines of the Administrative Panel on Laboratory Animal Care of China. Under general intraperitoneal anesthesia and after disinfection of the back of each of the 24 mice, two subcutaneous pockets were bluntly created through a $1.5-\mathrm{cm}$ incision in the back. A construct (induced cell-PLGA construct or non-induced cell-PLGA construct control) was inserted in each subcutaneous pocket. The wound was closed using a single interrupted suture. No animal died during the experimental period. The animals were sacrificed and the implants were harvested at 8 and 12 weeks, respectively.

Histological and immunohistochemical analyses. Eight and 12 weeks after implantation, the implants were retrieved and analyzed histologically and immunohistochemically, respec- tively. For histological analyses, specimens were fixed in $10 \%$ $(\mathrm{v} / \mathrm{v})$ buffered formalin, dehydrated with a series of graded alcohol and embedded in paraffin. Tissue sections $(4-\mu \mathrm{m}$ thick) were stained with hematoxylin and eosin for morphological analysis.

Expression of collagen type II was detected using monoclonal antibodies (Dako). Briefly, after deparaffinization, sections were predigested with trypsin at $37^{\circ} \mathrm{C}$ for $30 \mathrm{~min}$ to facilitate antibody access, endogenous peroxidase was removed by the treatment of $0.3 \% \mathrm{H}_{2} \mathrm{O}_{2}$ in methanol at room temperature for $30 \mathrm{~min}$, and non-specific antibody binding was blocked by incubation of sections in $10 \%$ normal goat serum at $37^{\circ} \mathrm{C}$ for $30 \mathrm{~min}$. Mouse anti-canine collagen type II diluted 1:100 in 0.01 M PBS (pH 7.4) was applied as a primary antibody at $4{ }^{\circ} \mathrm{C}$ overnight. Sections were then incubated with the secondary antibody, rabbit anti-mouse immunoglobulin (Dako) for $60 \mathrm{~min}$, followed by application of mouse PAP kit (Dako). Collagen type II was visualized by the reactions with $0.05 \%$ diaminobenzidine containing $0.01 \% \mathrm{H}_{2} \mathrm{O}_{2}$.

Analysis of mRNA for extracellular matrices and collagen with reverse transcriptase-polymerase chain reaction. RNA samples were obtained after 12 weeks and transcribed into cDNA by real-time reverse transcriptase-polymerase chain reaction (RT-PCR) for gene expression of type I collagen, type II collagen and aggrecan. After explantation, the constructs were digested with Ultraturax ${ }^{\mathrm{TM}}$ and total mRNA was prepared using TRIzol reagent according to the manufacturer's instructions (Gibco-BRL). Total RNA ( $1 \mu \mathrm{g})$ was treated with 1 unit of deoxyribonuclease I (DNase I; Gibco-BRL) in order to digest genomic DNA contamination. Random-primed cDNA synthesis was performed using $1 \mu \mathrm{g}$ of DNase I-treated total RNA and 50 units of StrataScript reverse transcriptase according to the manufacturer's instructions (Stratagene, La Jolla, CA, USA). TaqMan ${ }^{\text {TM }}$ PCR assays were performed in 96-well optical plates on an ABI Prism 7700 Sequence Detection system (Applied Biosystems, Forster City, CA, USA) using ABsolute QPCR ROX Mix (Abgene, Hamburg, Germany) according to the manufacturer's instructions. The thermal cycling conditions were $95^{\circ} \mathrm{C}$ for $15 \mathrm{~min}$ followed by 40 cycles at $95^{\circ} \mathrm{C}$ for $15 \mathrm{sec}$ and $60^{\circ} \mathrm{C}$ for $1 \mathrm{~min}$. The PCR products were analyzed by electrophoresis in $2 \%$ agarose gels and stained with ethidium bromide. The mRNAs analyzed were collagen I (681 bp), collagen II (447 bp), aggrecan (321 bp) and glyceraldehyde-3-phosphate dehydrogenase (GAPDH) (211 bp). Primer sequences for GAPDH, aggrecan, collagen I and collagen II were as follows: GAPDH, sense 5'-CCTCTATGCCAACACAGTGC-3' and antisense 5'-GTACTCCTGCTTGCTGATCC-3'; aggrecan, sense 5'-TAG AGAAGAAGAGGGGTTAGG-3' and antisense 5'-AGCACT AGGAGCCAGGGTTAT-3'; collagen I, sense 5'-ATGCCC AAGACTACCAGTGG-3' and antisense 5'-TCCTGG AAGCTCTTCTCAGT-3'; collagen II, sense 5'-TTTCCC AGGTCAAGATGGTC-3' and antisense 5'-CTTCAGCAC CTGTCTCACCA-3'.

Biomechanical analysis of engineered cartilage. Eight and 12 weeks after implantation, the biomechanical properties of the engineered cartilage were tested by measuring the compressive modulus. Briefly, the specimens were harvested and trimmed 

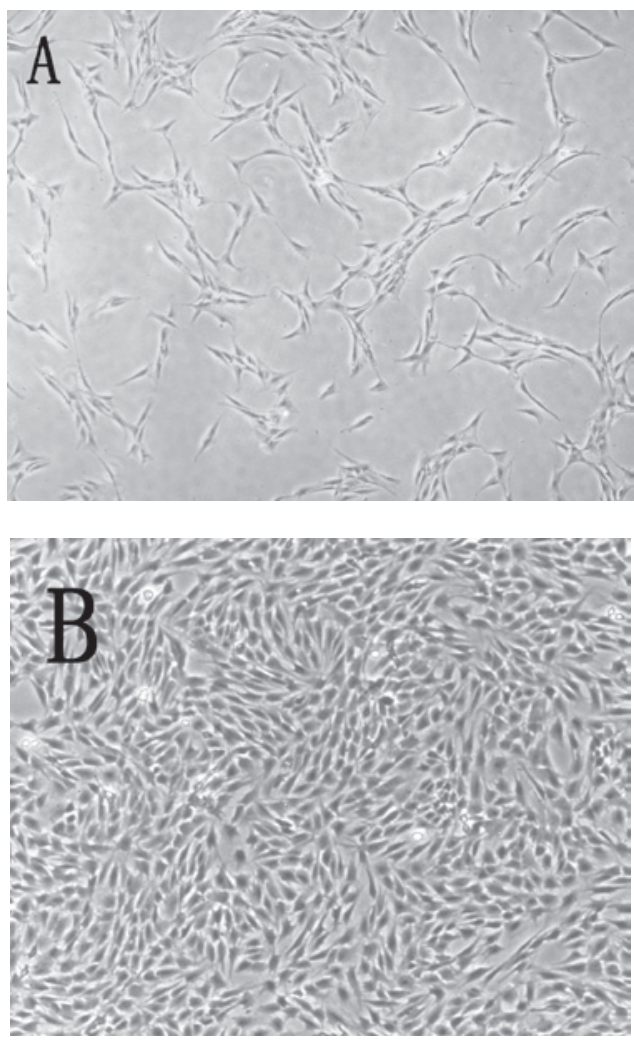

Figure 1. Morphological change between primary myoblasts and chondrogenically induced myoblasts. (A) Morphology of canine myoblasts cultured in a monolayer manner on day 3 in primary culture. (B) Myoblasts undergo chondrogenic differentiation after 14 days. Magnification, $100 \mu \mathrm{m}$.

to fit in a test chamber ( $5 \mathrm{~mm}$ diameter) of a biomechanical analyzer (Instron, Canton, MA, USA). A constant compressive strain rate of $1 \mathrm{~mm} / \mathrm{min}$ was used until the maximal force of $450 \mathrm{~N}$ was reached, and thus a force-displacement curve was obtained. The compressive moduli of tested tissues were automatically calculated by the machine and further verified by manual calculation with the formula: $\Delta P / A \times L / \Delta L(\Delta P$, the compressive force margin of the 2 points on the linear segment of the load-displacement curve before the first break point; $\Delta L$, the displacement margin of the corresponding two abovementioned points; $A$, the area of tested tissue; $L$, the thickness of tested tissue). In addition, the normal canine menisci were tested as a control. ANOVA analysis was applied and $\mathrm{P}<0.05$ was considered to indicate a statistically significant result.

Glycosaminoglycan (GAG) quantification of engineered cartilage. GAG quantitative analysis of 8- and 12-week specimens was performed. Briefly, the specimens were minced and triturated to prepare the protein solution. A series of reagents was added step by step to ensure the specific binding of Alcian blue and polysulfated molecules of GAGs in engineered cartilage. All GAGs were precipitated specifically in guanidine- $\mathrm{HCl}$ by using a low $\mathrm{pH}$ in combination with detergent and a high salt concentration. The precipitate was dissolved in a mixture of guanidine- $\mathrm{HCl}$ and propanol. For quantification, the absorbance was recorded in a microplate reader with a 600-nm filter, and a linear standard curve between 0.5 and $20 \mu \mathrm{g}$ was generated by adding known amounts of proteoglycans. The results were
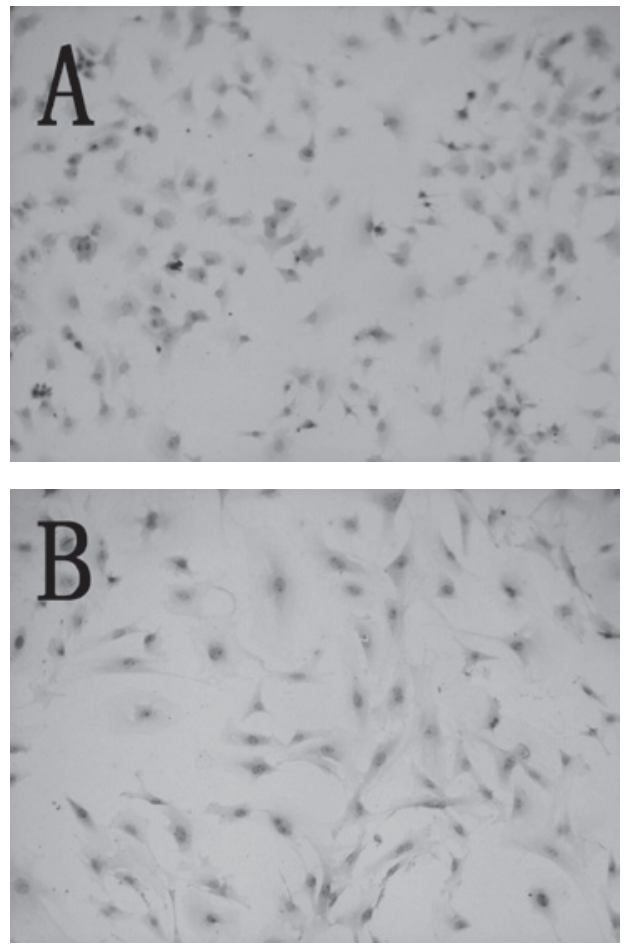

Figure 2. Immunocytochemical staining demonstrates a stronger expression of collagen II in (A) induced cells than in (B) non-induced cells. Magnification, $200 \mu \mathrm{m}$.

analyzed using the ANOVA test. A P-value $<0.05$ was considered statistically significant. Since no neocartilage was generated we did not analyse the quantitive GAG in the control group. The positive control was also tested as a normal meniscus.

Statistical analysis. The presence of type II collagen in the repaired meniscus tissue from the different groups was noted and statistically compared using the Fisher's exact test. Compressive modulus and GAG content in the different groups were analyzed with ANOVA. For all evaluations, the level of statistical significance was set at a probability value of $<0.05$.

\section{Results}

Morphological and immunohistochemical analysis. As shown in Fig. 1, myoblasts induced with CDMP-2 and TGF- $\beta 1$ underwent a morphological change after chondrogenic induction, approaching the shape of native chondrocytes. In addition, the induced cells showed significantly enhanced collagen II expression compared to control cells (Fig. 2).

Gross morphology of engineered cartilage. Specimens harvested after 8 weeks of in vivo culture showed that all induced cell-PLGA constructs maintained the approximate original scaffold shape and size, and had a compact consistency (Fig. 3). The non-induced cell-PLGA constructs showed a failure to maintain the shape and size of the original scaffold. Twelve-week specimens showed a more cartilaginous appearance. However, no construct was identified in the control group, indicating that the scaffold was degraded completely at 12 weeks. 

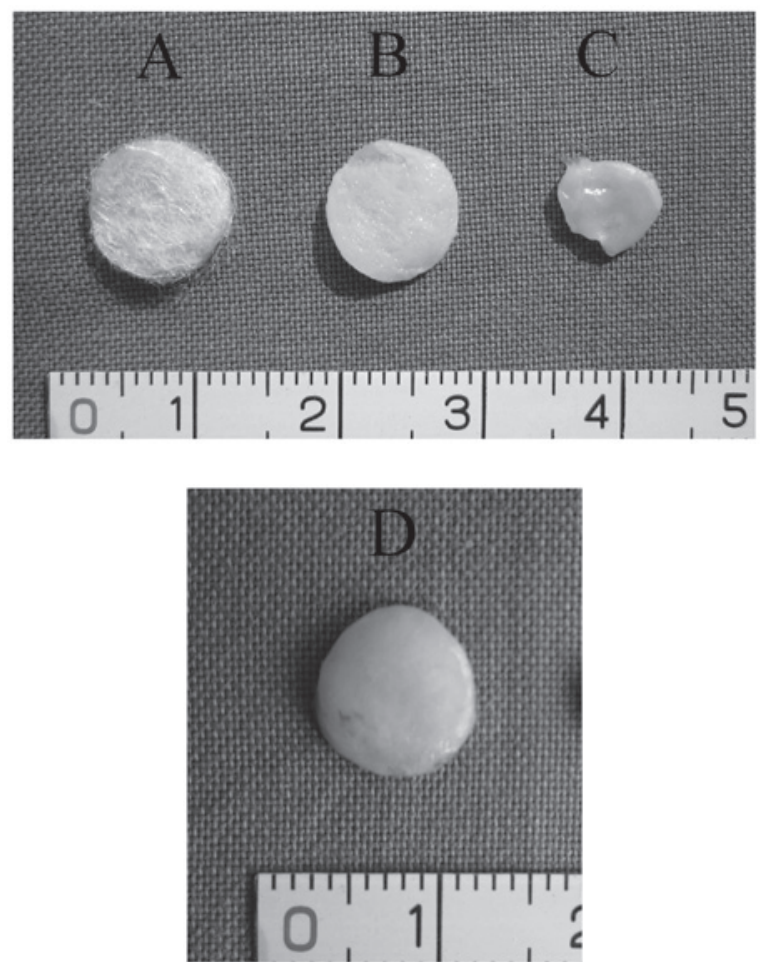

Figure 3. Gross morphology of engineered cartilage. (A) A PLGA scaffold, (B) an induced cell-PLGA construct at 8 weeks post-implantation, (C) a non-induced cell-PLGA construct at 8 weeks post-implantation and (D) an induced cell-PLGA construct at 12 weeks post-implantation. PLGA, polylactide-co-glycolide.

Histological and immunohistochemical assessment of engineered cartilage. A histological section of engineered cartilage at 8 weeks had the appearance of fibrocartilage with fibrochondrocytic-like cells (Fig. 4A). In the control group, only a band of fibrous tissue was observed (Fig. 4B). The engineered cartilage at 12 weeks had histological structures more similar to those of normal cartilage than those at 8 weeks (Fig. 4C).

Immunohistological staining indicated that engineered cartilage was positively stained for type II collagen at 8 weeks post-implantation (Fig. 5A). With the maturation of engineered cartilage, the expression and distribution of collagen II was found to be similar to that of the neighboring native meniscus at 12 weeks post-implantation (Fig. 5C). However, no expression of collagen II was observed in all specimens of the control group at 8 weeks (Fig. 5B).

Expression of cartilage-specific genes by RT-PCR. To investigate the chondrogenic differentiation of the engineered cartilage, mRNA expression of type I collagen, type II collagen and aggrecan was assessed using RT-PCR. At 12 weeks, expression of mRNAs for type I collagen, type II collagen and aggrecan was detected. By contrast, none of the assessed genes were expressed in the control tissue (Fig. 6). Gene expression of the normal meniscus was assessed as a positive control.

Biomechanical properties of engineered cartilage. As summarized in Table I, the compressive moduli increased with length of transplantation time. At 8 weeks, the moduli reached
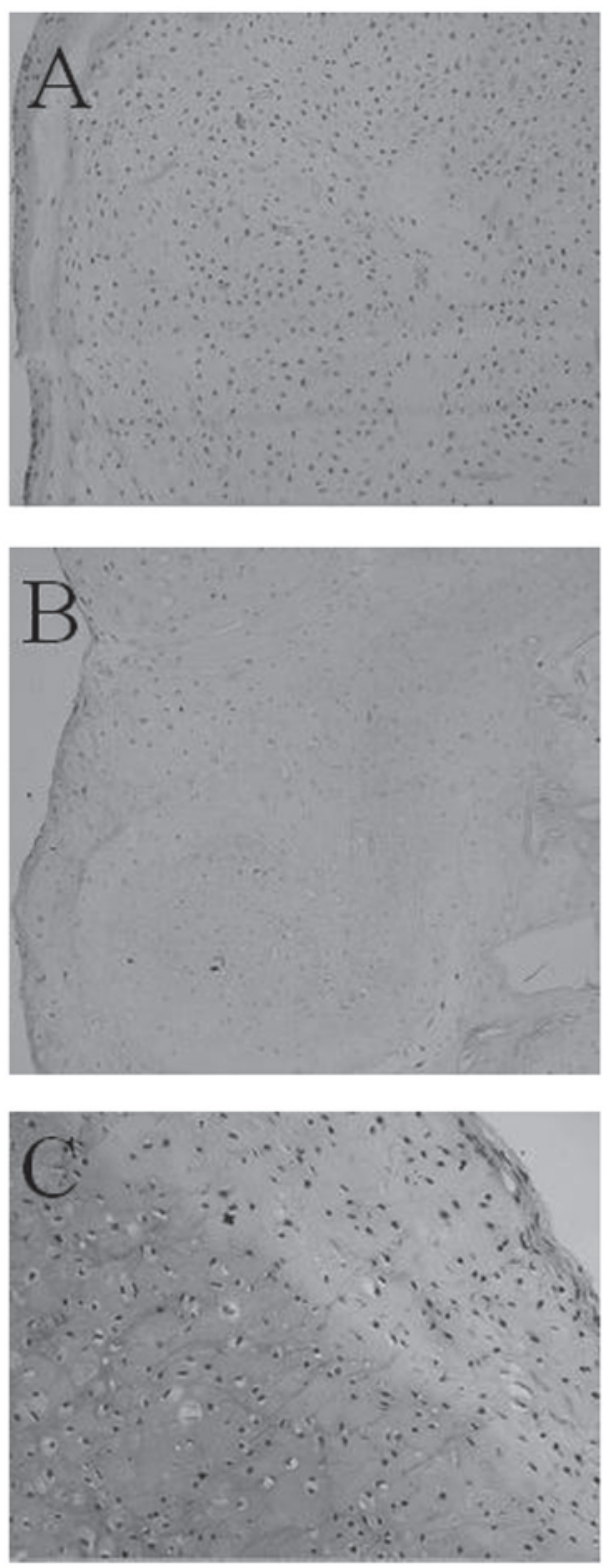

Figure 4. Hematoxylin and eosin histology of engineered cartilage. (A) An induced cell-PLGA construct at 8 weeks post-implantation, (B) a noninduced cell-PLGA construct at 8 weeks post-implantation and (C) an induced cell-PLGA construct at 12 weeks post-implantation. PLGA, polylactide-co-glycolide.

50.41 and $23.16 \%$ of the normal meniscus level, respectively, in the experimental and control groups, and the experimental group increased further to $85.72 \%$ at 12 weeks. Statistical analysis demonstrated that the compressive modulus was higher in the induced group (Exp) than in the non-induced group $(\mathrm{Ctrl})(\mathrm{P}<0.05)$, indicating that in vitro chondrogenic induction is helpful for improving biomechanical properties of myoblast-engineered cartilage.

GAG content quantification. The GAG deposition of the engineered cartilage was further quantified by biochemical analysis and also compared with that of the normal meniscus. As shown in Table II, it was found that the amount of GAG in the experimental group at 8 weeks reached $47.39 \%$ of that in the normal meniscus, as compared with $23.26 \%$ for 
Table I. The compressive moduli of repaired cartilage.

\begin{tabular}{|c|c|c|c|c|}
\hline \multirow[b]{2}{*}{ Groups } & \multicolumn{2}{|c|}{8 weeks $(n=12)$} & \multicolumn{2}{|c|}{12 weeks $(n=12)$} \\
\hline & Moduli (MPa) & Percentage of normal (\%) & Moduli (MPa) & Percentage of normal (\%) \\
\hline Normal & $27.12 \pm 0.69$ & & $28.92 \pm 1.35$ & \\
\hline Exp & $13.67 \pm 1.830$ & $50.41 \pm 2.93$ & $24.79 \pm 2.78$ & $85.72 \pm 4.23$ \\
\hline Ctrl & $6.28 \pm 1.52$ & $23.16 \pm 2.49$ & & \\
\hline
\end{tabular}

The compressive moduli increased with the transplanted time and the value is always higher in induced cell-PLGA constructs group (Exp) than in non-induced cell-PLGA constructs group $(\mathrm{Ctrl})$ with a statistically significant difference $(\mathrm{P}<0.05)$.
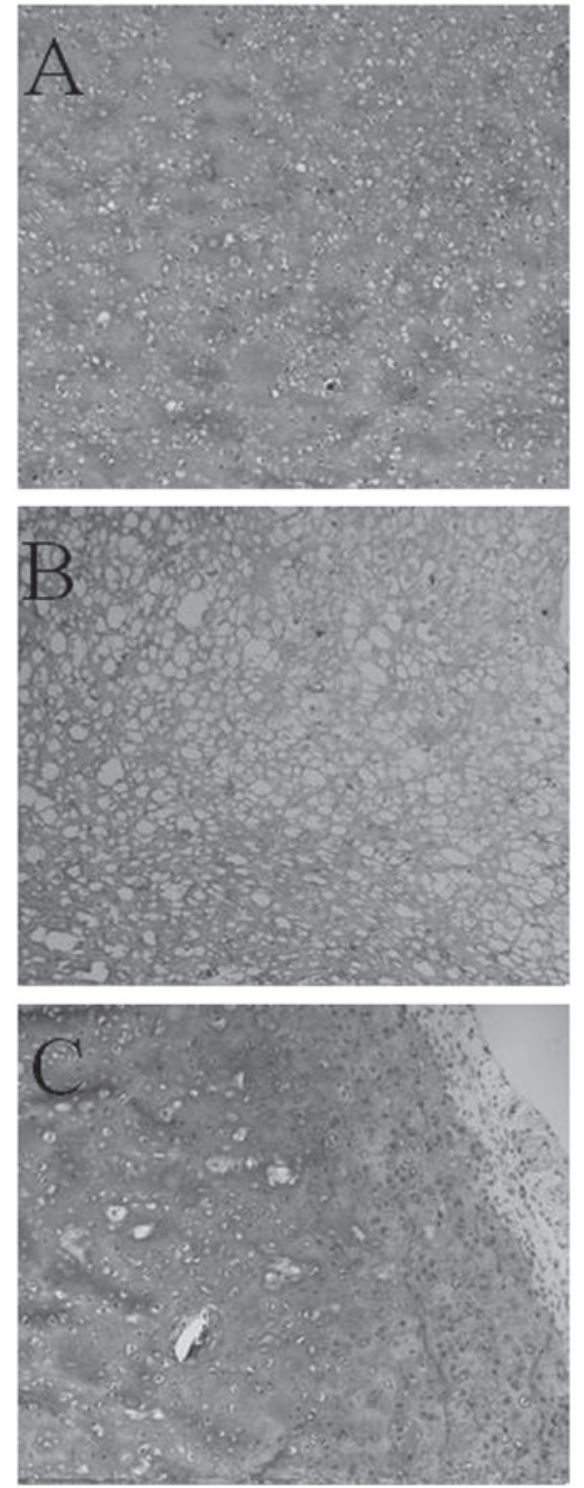

Figure 5. Type II collagen immunohistochemistry of engineered cartilage. (A) An induced cell-PLGA construct at 8 weeks post-implantation, (B) a non-induced cell-PLGA construct at 8 weeks post-implantation and (C) an induced cell-PLGA construct at 12 weeks post-implantation. PLGA, polylactide-co-glycolide.

the control group $(\mathrm{P}<0.05)$. However, with the maturation of the engineered cartilage to 12 weeks, GAG content was increased, reaching $91.35 \%$ of that in the normal meniscus

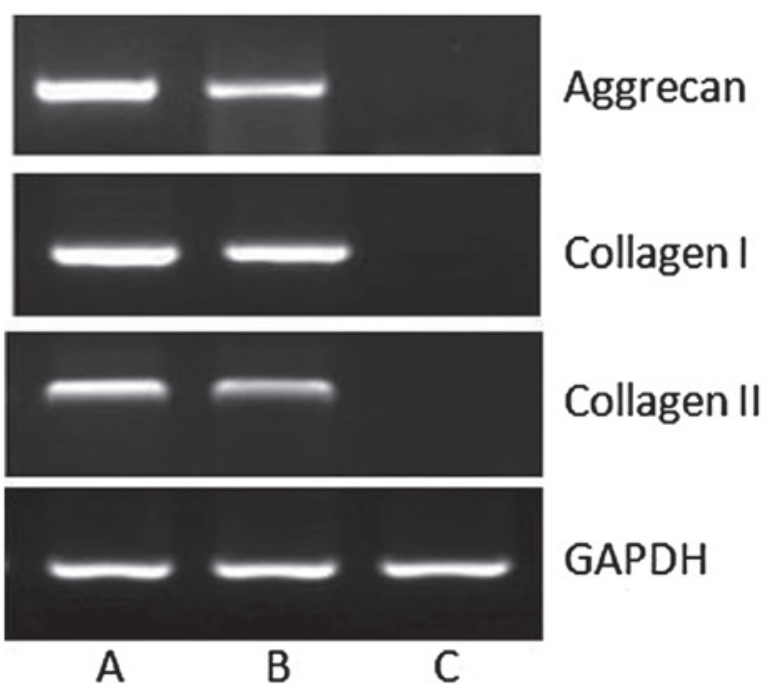

Figure 6. Analysis of expression of mRNA for aggrecan, collagen I, II and GAPDH with RT-PCR. (A) Positive control using a normal meniscus. (B) An induced cell-PLGA construct 12 weeks post-implantation and (C) a non-induced cell-PLGA construct 12 weeks post-implantation. PLGA, polylactide-co-glycolide.

$(\mathrm{P}<0.05)$. A significant difference was also observed between the amount of GAG in the engineered cartilage at 8 and 12 weeks $(\mathrm{P}<0.05)$.

\section{Discussion}

Meniscal injury is one of the most common injuries to the knee. The menisci are important for normal knee function. A meniscus injury increases the risk of subsequent development of degenerative changes in the knee (11). Repairing meniscus injuries remains a challenge in sports medicine, since meniscus usually does not regenerate by itself following injury. The emergence of a tissue engineering technique may provide a satisfactory solution to the problem.

Tissue engineering is based on a unique combination of exogenous cells, specific stimuli and matrix scaffold in an in vitro or in vivo environment. In the present study, myoblasts were used. Myoblasts, which are adult stem cells and include skeletal muscle cell precursors, have been shown to possess multipotential mesenchymal stem cell activity and are capable of forming chondrocytes, osteocytes and adipocytes as well as myocytes (12-14). Previous reports suggest that 
Table II. GAG contents (wet weight) of repaired cartilage.

\begin{tabular}{|c|c|c|c|c|}
\hline \multirow[b]{2}{*}{ Groups } & \multicolumn{2}{|c|}{8 weeks $(n=12)$} & \multicolumn{2}{|c|}{12 weeks $(n=12)$} \\
\hline & GAG content $(\mathrm{mg} / \mathrm{g})$ & Percentage of normal (\%) & GAG content $(\mathrm{mg} / \mathrm{g})$ & Percentage of normal (\%) \\
\hline Normal & $12.07 \pm 0.37$ & & $12.95 \pm 1.23$ & \\
\hline Exp & $5.72 \pm 0.87$ & $47.39 \pm 2.37$ & $11.83 \pm 3.05$ & $91.35 \pm 5.27$ \\
\hline Ctrl & $2.81 \pm 1.03$ & $23.26 \pm 4.76$ & & \\
\hline
\end{tabular}

a degree of plasticity remains prior to terminal myoblastic differentiation. Therefore, myoblasts may prove useful for the development of new therapeutic approaches aimed at the regeneration of damaged or diseased tissues. Myoblasts have been investigated as a candidate cell source for tissue engineering (15-17). In comparison with other stem cell sources, myoblasts represent a more promising source for cartilage engineering, as they are relatively abundant and easily accessible, with minimal donor site morbidity $(18,19)$. Studies have shown that myoblasts have a higher cell yield and more rapid proliferation ability during in vitro expansion $(8,20)$. In this study, we observed a chondrogenic response with a dose of $50 \mathrm{ng} / \mathrm{ml}$ CDMP- 2 and $20 \mathrm{ng} / \mathrm{ml}$ TGF- $\beta 1$ provided continuously with each medium change. Chondrogenic differentiation, analyzed using immunohistochemistry and gene expression profiles, was observed. This suggests that key events responsible for the commitment of myoblasts to the chondrogenic lineage occur during the early initial period of cell growth and proliferation.

PGA is a commonly used synthetic polymer in cartilage tissue engineering. In order to maintain its dimensional stability and enhance its mechanical properties, fibrous PGA meshes are coated with solutions of PLA. Evaporation of the solvent for PLA leads to the formation of PLGA composites with specific shapes. The feasibility of using a PLGA composite as a scaffold to engineer cartilage tissue has been documented in a number of studies $(21,22)$. It was also shown that adhesion and proliferation of chondrocytes on PGA fibers was significantly suppressed when an increased amount of PLA was added $(21,22)$. Therefore, the concentration of PLA solution to be added needs to be lowered but still sufficient to function as glue to maintain the structural stability of the PGA 3D scaffold. In the present study, 1.5\% PLA in dichloromethane was used. Any further lowering would cause an unstable configuration of the scaffold. Scanning electron microscopy revealed that PLA at this concentration is capable of wrapping PGA fibers together and that the shape of scaffold was maintained when they were kept in culture medium for as long as 5 weeks (22).

The main advantage of using a PLGA scaffold for meniscal tissue engineering is its suitable degradation rate, which matches the kinetics of new meniscal formation in vivo (5). The degradation of non-woven PGA scaffold is reported to be complete over a period of 2 months in vivo (23). In the present study, no undegraded PLGA fibers were observed histologically in the experimental or control groups at 12 weeks post-implantation. Due to its rapid degradation, PLGA scaffold was found to accelerate chondrogenesis of constructs prepared from dedifferentiated chondrocytes and PLGA, as the accumulation of the deposited cartilagespecific extracellular matrix (ECM) and expression of marker genes both in vitro and in vivo were significantly enhanced compared with those of constructs prepared from PGA. It was also proposed that early degradation of PLGA fibers may have a positive effect on chondrogenesis by leaving new spaces for cells to further fill in and produce new intercellular matrix, which in turn may facilitate the formation of more cell-matrix and cell-cell contact.

In this study, the specimens showed a cartilaginous appearance. However, no construct was identified in all control groups after 8 weeks of in vivo culture. As shown by the histological analysis, the section of engineered cartilage had the appearance of fibrocartilage with fibrochondrocytic-like cells at 8 and 12 weeks. In the control group, only a band of fibrous tissue was observed. Immunohistological staining indicated that engineered cartilage was positively stained for type II collagen compared with the control group at 8 and 12 weeks post-implantation. At 12 weeks, expression of mRNAs for type I collagen, type II collagen and aggrecan was detected by RT-PCR. By contrast, no assessed genes were expressed in the control tissue. In addition, the biomechanical properties (compressive modulus) and biochemical composition (GAG quantification) of engineered cartilage at 12 weeks postimplantation were similar to those of corresponding normal cartilage, which indicated that the engineered cartilage was formed both structurally and functionally.

In conclusion, we showed chondrogenic differentiation of myoblasts seeded into PLGA scaffolds following implantation in a subcutaneous pocket of nude mice. Further studies are required to investigate implanted myoblast-seeded PLGA scaffolds in a joint, including biomechanical stress for the evaluation of a possible positive stimulus by mechanical loading after implantation.

\section{Acknowledgements}

This study was supported by the Shanghai Natural Science Foundation (Grant No. 09ZR1425500) and Research Grant for Health Science and Technology of Pudong Health Bureau of Shanghai (Grant No. PW2009B-2). 


\section{References}

1. Hart R, Janecek M, Siska V, Kucera B and Stipcák V: Correlation of long-term clinical and radiological results after meniscectomies. Acta Chir Orthop Traumatol Cech 72: 304-307, 2005.

2. van der Wal RJ, Thomassen BJ and van Arkel ER: Long-term clinical outcome of open meniscal allograft transplantation. Am J Sports Med 37: 2134-2139, 2009.

3. Yamasaki T, Deie M, Shinomiya R, Yasunaga Y, Yanada S and Ochi M: Transplantation of meniscus regenerated by tissue engineering with a scaffold derived from a rat meniscus and mesenchymal stromal cells derived from rat bone marrow. Artif Organs 32: 519-524, 2008.

4. Webber RJ, York JL, Vanderschilden JL and Hough AJ Jr: An organ culture model for assaying wound repair of the fibrocartilaginous knee joint meniscus. Am J Sports Med 17: 393-400, 1989.

5. Kang SW, Son SM, Lee JS, Lee ES, Lee KY, Park SG, Park JH and Kim BS: Regeneration of whole meniscus using meniscal cells and polymer scaffolds in a rabbit total meniscectomy model. J Biomed Mater Res A 78: 659-671, 2006.

6. Ishida K, Kuroda R, Miwa M, Tabata Y, Hokugo A, Kawamoto T, Sasaki K, Doita M and Kurosaka M: The regenerative effects of platelet-rich plasma on meniscal cells in vitro and its in vivo application with biodegradable gelatin hydrogel. Tissue Eng 13: 1103-1112, 2007.

7. Peretti GM, Gill TJ, Xu JW, Randolph MA, Morse KR and Zaleske DJ: Cell-based therapy for meniscal repair: a large animal study. Am J Sports Med 32: 146-158, 2004.

8. Lu SH, Yang AH, Wei CF, Chiang HS and Chancellor MB: Multi-potent differentiation of human purified muscle-derived cells: potential for tissue regeneration. BJU Int 105: 1174-1180, 2009.

9. Zhou G, Liu W, Cui L, Wang X, Liu T and Cao Y: Repair of porcine articular osteochondral defects in non-weightbearing areas with autologous bone marrow stromal cells. Tissue Eng 12: 3209-3221, 2006.

10. Zhu W, Wang Y, Qiu G and Chen B: Characterization of the purification and primary culture of adult canine myoblasts in vitro Mol Med Report 3: 463-468, 2010.

11. Gu YL and Wang YB: Treatment of meniscal injury: a current concept review. Chin J Traumatol 13: 370-376, 2010.
12. Gu Y, Wang Y, Dai H, Lu L, Cheng Y and Zhu W: Chondrogenic differentiation of canine myoblasts induced by cartilage-derived morphogenetic protein-2 and transforming growth factor- $\beta 1$ in vitro. Mol Med Report 5: 767-772, 2012.

13. Asakura A, Komaki M and Rudnicki M: Muscle satellite cells are multipotential stem cells that exhibit myogenic, osteogenic and adipogenic differentiation. Differentiation 68: 245-253, 2001.

14. Matsushita T, Matsui N, Fujioka H, Kubo S, Kuroda R, Kurosaka M and Yoshiya S: Expression of transcription factor sox9 in rat L6 myoblastic cells. Connect Tissue Res 45: 164-173, 2004.

15. Goldring K, Partridge T and Watt D: Muscle stem cells. J Pathol 197: 457-467, 2002.

16. Day CS, Kasemkijwattana C, Menetrey J, Floyd SS Jr, Booth D, Moreland MS, Fu FH and Huard J: Myoblast-mediated gene transfer to the joint. J Orthop Res 15: 894-903, 1997.

17. Koning M, Harmsen MC, van Luyn MJ and Werker PM: Current opportunities and challenges in skeletal muscle tissue engineering. J Tissue Eng Regen Med 3: 407-415, 2009.

18. Singh D, Nayak V and Kumar A: Proliferation of myoblast skeletal cells on three-dimensional supermacroporous cryogels. Int J Biol Sci 6: 371-381, 2010.

19. Marsano A, Millward-Sadler SJ, Salter DM, Adesida A, Hardingham T, Tognana E, Kon E, Chiari-Grisar C, Nehrer S, Jakob M and Martin I: Differential cartilaginous tissue formation by human synovial membrane, fat pad, meniscus cells and articular chondrocytes. Osteoarthritis Cartilage 15: 48-58, 2007.

20. Stern-Straeter J, Bran G, Riedel F, Sauter A, Hörmann K and Goessler UR: Characterization of human myoblast cultures for tissue engineering. Int J Mol Med 21: 49-56, 2008.

21. Moran JM, Pazzano D and Bonassar LJ: Characterization of polylactic acid-polyglycolic acid composites for cartilage tissue engineering. Tissue Eng 9: 63-70, 2003.

22. Cui L, Wu Y, Cen L, Zhou H, Yin S, Liu G, Liu W and Cao Y: Repair of articular cartilage defect in non-weight bearing areas using adipose derived stem cells loaded polyglycolic acid mesh. Biomaterials 30: 2683-2693, 2009.

23. Zwingmann J, Mehlhorn AT, Südkamp N, Stark B, Dauner M and Schmal H: Chondrogenic differentiation of human articular chondrocytes differs in biodegradable PGA/PLA scaffolds. Tissue Eng 13: 2335-2343, 2007. 